intervention was gradually implemented over time through increasing awareness and engagement with education and exposure to the project. Logistical issues, such as remembering to offer a test, were overcome with practical facilitators like computer alerts. However, integration was limited as not all GPs utilised the intervention components or other clinic staff to increase testing because of restrictions in clinic structure.

Conclusion GPs reported that the ACCEPt intervention and its implementation within the NPT framework have been effective at facilitating chlamydia testing in general practice.

Disclosure of interest statement ACCEPt was commissioned and funded by the Australian Government Department of Health and Ageing. Additional funding has been received from the National Health and Medical Research Council, the Victorian Department of Health and NSW Health.

\section{P04.17 INTRAVAGINAL PRACTICES AMONG RURAL MALAWIAN WOMEN}

${ }^{1}$ A Esber*, ${ }^{2}$ A Norris Turner, ${ }^{1}$ A Norris. ${ }^{1}$ Division of Epidemiology, College of Public Health, The Ohio State University; ${ }^{2}$ Divison of Infectious Diseases, College of Medicine, The Ohio State University

10.1136/sextrans-2015-052270.271

Introduction Intravaginal practices (IVP) are highly prevalent and commonly performed in many countries for a variety of purposes related to genital health, personal hygiene, and sexual pleasure. However, IVP may also have harmful side effects. Previous research supports an association between IVP and bacterial vaginosis and HIV. Our objective is to understand the prevalence and motivations for IVP among rural Malawian women participating in a community survey on sexual and reproductive health.

Methods We used baseline survey data from a community-based cohort study conducted among 650 women in rural Lilongwe District, Malawi. Participants answered questions assessing frequency of use for different types of IVP and reasons for performing IVP.

Results Most women reported at least some experience with IVP in the past 30 days: $88 \%$ reported internal vaginal cleansing with water, $87 \%$ reported cleansing with soap and water, and $84 \%$ reported inserting cotton, cloth or tissue. Only 5\% of women reported no IVP; most (60\%) reported at least three practices. Approximately half of women reported very frequent engagement in at least one type of IVP: among those who inserted cotton, cloth or tissue, $43 \%$ did so more than once a day; among those who cleansed internally with soap and water, $51 \%$ did so more than once a day. Among IVP engagers, 57\% reported sexual- and hygiene-related reasons, $36 \%$ only hygiene reasons, $4 \%$ neither, and 3\% only sexually-related motivations. We found no significant association between motivations for IVP (for hygiene vs. sexual reasons) and frequency of IVP.

Conclusion Intravaginal practices are highly prevalent and frequently performed among these rural Malawian women. Women's motivations for IVP were not associated with IVP frequency, and therefore IVP cessation programs targeted to motivations may be unsuccessful in this population. The next phase of this research program will investigate the association between IVP and STI prevalence.

Disclosure of interest statement The authors have no conflicts of interest or financial disclosures to report.
P04.18 ARE INTRAVAGINAL PRACTICES ASSOCIATED WITH PRECANCEROUS LESIONS AND HPV INFECTION?

${ }^{1}$ A Esber*, ${ }^{1}$ A Norris, ${ }^{2}$ AN Turner. ${ }^{1}$ Division of Epidemiology, College of Public Health, The Ohio State University; ${ }^{2}$ Divison of Infectious Diseases, College of Medicine, The Ohio State University

\subsection{6/sextrans-2015-052270.272}

Introduction Many women engage in intravaginal practices (IVP) to improve genital hygiene and sexual pleasure. However, IVP can disrupt the genital mucosa, possibly increasing acquisition risk of HIV and the reproductive tract infection bacterial vaginosis. Limited prior research also suggests an association between IVP and HPV. In this analysis, we examine associations between IVP, precancerous lesions and high risk HPV (hr-HPV).

Methods At a rural clinic in Lilongwe District, Malawi, we began enrolling women in a cross-sectional study in January 2015. As of end of March, 96 women have been enrolled and partial results are available. Enrollment is expected to conclude by May 2015; hr-HPV testing to be complete by June 2015. Eligible women are aged 18 and older and presenting at the clinic with genitourinary symptoms. All women undergo visual inspection of the cervix with acetic acid (VIA) and clinician-collected cervical sampling for hr-HPV testing. Women also answer a questionnaire capturing the type and frequency of IVP.

Results IVP were commonly reported among participants: 97\% reported cleansing the vagina with cotton, cloth or tissue; $44 \%$ cleansed with soap and water; and $8 \%$ inserted alum or other powder, herbs, leaves, castor oil or traditional products. IVP was also very frequent, with $85 \%$ of women reporting at least one practice daily. Twelve women (15\%) had abnormal lesions identified during VIA. We observed no significant association between frequency or type of IVP and the presence of abnormal lesions.

Conclusion Our data confirm that intravaginal practices are common in Malawi among care-seeking women. As our sample size increases, any associations will become more precise. If IVP is associated with abnormal cervical lesions or hr-HPV prevalence, these practices could represent a modifiable risk factor to target in future health interventions in this region.

Disclosure of interest statement The authors have no conflicts of interest or financial disclosures to report.

\section{P04.19 ALCOHOL INVOLVEMENT IN SEXUAL BEHAVIOUR AND ADVERSE SEXUAL HEALTH OUTCOMES IN EARLY MIDDLE AGE}

JL Connor ${ }^{*}, \mathrm{R}$ Kydd, N Dickson. Department of Preventive and Social Medicine, University of Otago, Dunedin, New Zealand

10.1136/sextrans-2015-052270.273

Introduction Research on alcohol and sexual behaviour has focused on young adults or high-risk groups, showing alcohol use contributing to riskier sexual choices. Adults now in early midlife have been exposed to heavier drinking norms than previously, raising questions about effects on sexual well-being. We examined self-reported use and consequences of alcohol in sexual contexts, and its association with usual drinking pattern at age 38, and also associations of heavy drinking occasion (HDO) frequency with number of sexual partners, sexually transmitted infection (STI), and termination of pregnancy (TOP), from 2632 and 32-38 years of age.

Methods Members of the Dunedin Study birth cohort answered computer-presented questions about sexual behaviour and 